\title{
PARTICLE MODELS AND NONCOMMUTATIVE GEOMETRY
}

\author{
Alain CONNES \\ IHES, 35 route de Chartres, Bures-sur-Yvette, F-91440 FRANCE \\ John LOTT \\ Department of Mathematics, University of Michigan. Ann Arbor, MI 48109, USA
}

We write three particle models in terms of noncommutative gauge theory: the Glashow-Weinberg-

Salam model, the Peccei-Quinn model and the standard model.

\section{Introduction.}

While quantum field theoretic models of pure Yang-Mills type are appealing both physically and geometrically (as the Yang-Mills action hạs a clear geometric significance) they are unphysical since they only give rise to massless vector bosons. In order to circumvent this problem one adds new fields, the Higgs fields, with a symmetry-breaking mechanism which provides masses for botil the vector bosons and the fermions. Our aim in this paper is show that at the expense of modifying the usual notions of a geometric spacetime, one can recover several mociels of particle physics, all involving Higgs fields, as pure YangMills models.

It gradually emerges from problems in pure mathematics [Co1] that the class of Riemannian metric spaces is ton narrow to encompass some interesting spaces, and that to do so one must reformulate notiors of geometry in operator algebraic terms. The basic objects of such a geometry are a (possibly noncommutative) algebra $\mathbf{a}$, a Hilbert space on which the algebra acts and an operator on the Hilbert space. The algebra can be considered to be generalizing the idea of a manifold, while the operator provides the metric structure. (One finds usual Riemannian geometry as a special case when a manifold $M$ is replaced by its commutative algebra $a$ of functions, the Hilbert space is that of spinors on $\mathrm{M}$ and the Riemannian metric is replaced by the Dirac operator.) It is then possible to write an action functional on operafor-theoretically defined gauge potentials. In the special case of an ordinary Riemannian manifold, the action functional reproduces the standard pure Yang-Mills action.

One can consider doing gauge theory on many types of spaces. (For example, instead of thinking of lattice gauge theory as a sinulation of the continuum theory, one can imagive doing a real gauge theory th the lattice.) While the original mathematical motivation was to encompass new situations in which the algebra $Q$ is no longer taken to be commutative, it has wider scope even wien the algebra is commutative i.e. when the algebra arises from an ordinary point set. This is because the generalized metric can be taken to be different from a usual metric, by using an operator different from the usual Dirac operator. As was shown in [Co2], a gauge fieid on an appropriate generalized Riemannian space consists of an ordinary gauge field and a Higgs field. The pure Yang-Mills action on the generalized space will decompose as the sum of the Yang-Mills action for the ordinary gauge field, the kinetic action for the Higgs field and a symmetry-breaking Higgs potential.

The underlying fields in noncommutative gauge theory are the spinor fields. The bosonic fields arise from representing the differential forms, which depend only on 
the algebra, on the Hilbert space. Thus to build our particle models we enter the phenomenological data of the fermion representations and the desired symmetry breaking. The fermion representations will determine which algebra we should use, and the vector bundles associated to the algebra. The desired symmetry breaking will determine how the generalized metric will differ from an ordinary metric. As the basic fields are fermionic, the fermionic action can be written in a straightforward way.

Our purpose is to recover models of particle physics by suitable choices of our generalized Riemannian metric space. This paper contains the results of calculations for specific models, along with their geometric interpretation. The outline is as follows. In section II we discuss noncommutative geometry and noncommutative gauge theory. In section III we give the Glashow-WeinbergSaiam (GWS) model for leptons. Our treatment is basically the same as for model II of [Co2], but we show that a defect of that model is corrected by the presence of several generations of leptons with different masses. The corresponding geometric space can be thought of as a spacetime of Kaluza-Klein type, where the fiber is formed of a two-point set. In section IV we add quarks to give the Peccei-Quinn model. The algebras of sections III and IV are both commutative. In section $\mathrm{V}$ we show that a quaternionic algebra yields the standard model. Section VI has a discussion of thè results.

We wish to emphasize that in this paper we only work at the classical level. Despite the appearance of Dirac operators, which sometimes have a quantum connotation, they are only used to define classical geometries.

J.L. wculd like to thank M. Berger and the IHES for their hospitality, and the Sloan Foundation for partial support.

\section{Noncommutative Gauge Theory.}

We will give a self-contained summary of what we need from noncommutative geometry. At the end of the section we give two point-by-point examples. The first is that of ordinary commutative geometry. The reader may want to follow this example concurrently with the definitions. The second is that of a two-point space. For more motivation and details, we refer to $[\mathrm{Co}, \mathrm{Co}]$ and references therein.

(1) A noncommutative space is given by a * algebra $a$ with unit.

Because $Q$ can be noncommutative, we will have to be careful in distinguishing between left and right actions. Given $a$, the algebra $M_{n}(a)$ of $n \times n$ matrices over $a$ forms another noncommutative space.

(2) If $\mathrm{Q}$ is a complex commutative algebra, define the character space $C$, or spectrum, of $Q$ to be the space of algebra homomorphisms from $\mathbb{C}$ to $\mathbb{C}$.

A right (left) module over $Q$ is a complex vector space on which $\mathrm{Q}$ acts on the right (or left). The tensor product $E^{\prime} \otimes_{a} \&$ of a left module $E^{\prime}$ and a right module $E$ is generated by

$\left\{\xi^{\prime} \otimes \xi_{:} \xi^{\prime} \in \mathcal{E}^{\prime}, \xi \in \xi^{\prime}\right\}$, with the relation

$\xi^{\prime} \mathrm{a} \otimes \xi-\xi^{\prime} \otimes \mathrm{a} \xi=0$. If $\notin$ and $\Im$ are right $\mathrm{Q}$-modules then we will write $\operatorname{End}_{Q}(\mathcal{E}, \mathcal{F})$ for

\{u: $\mathcal{E} \rightarrow \mathcal{F}, \mathrm{u}$ linear, $\mathrm{u}(\xi \mathrm{a})=\mathrm{u}(\xi) \mathrm{a}$ for all $\xi \in \mathcal{E}, \mathrm{a} \in$ a) and we will write $\operatorname{End}_{a}(\notin)$ for $\operatorname{End}_{a}(\ell, \ell)$.

(3) A vector bundle $\varepsilon$ associated to $a$ is a finite projective right module over $a$. This means that $\epsilon$ is the image $\mathrm{e}^{\mathrm{n}}$ of $\mathrm{a}^{\mathrm{n}}$ under some orthogonal projection

$e \in M_{n}(a)$.

The dual space $\varepsilon^{*}=\operatorname{End}_{G}(\varepsilon, Q)$ is a left module over $\mathcal{Q}$ with the $\mathcal{Q}$ action given by $(a \eta)(\xi)=a \eta(\xi)$ for all

$a \in a, \xi \in \mathcal{E}, \eta \in \mathcal{E}^{*}$. There is a pairing 
$():, \varepsilon^{*} \times \varepsilon \rightarrow a$ given by $(\eta, \xi)=\eta(\xi)$.

(4) The space of universal $k$-forms $\Omega^{k}(Q)$ is the bimodule generated by elements $\mathrm{a}_{0} \mathrm{da}_{1} \ldots \mathrm{da}_{\mathrm{k}}$,

with $a_{0}, a_{1}, \ldots, a_{k} \in Q$, and the relations

$d(a b)-(d a) b-a(d b)=0$ and $d(1)=0$. The space $\Omega^{*}(a)=$ $\oplus \Omega^{k}(a)$ of all differential forms is a graded differential algebra, with the differential given by

$d\left(a_{0} d a_{1} \ldots d a_{k}\right)=d a_{0} d a_{1} \ldots d a_{k}$. There is an involution on $\Omega^{*}(\mathcal{Q})$ given by

$\left(a_{0} d a_{1} \ldots d a_{k}\right)^{*}=d a_{k}^{*} \ldots d a_{1}^{*} a_{0} *$

(5) A hermitian metric on 6 is a map

$<,>: \bullet \times \bullet \rightarrow Q$ such that

1. $\left\langle\xi_{1} a_{1}, \xi_{2} a_{2}\right\rangle=a_{1}^{*}\left\langle\xi_{1}, \xi_{2}\right\rangle a_{2}$ for all $\xi_{1}, \xi_{2} \in G$. $a_{1}, a_{2} \in Q$

2. $\left\langle\xi_{1}, \xi_{2}\right\rangle^{*}=\left\langle\xi_{2}, \xi_{1}\right\rangle$ for all $\xi_{1}, \xi_{2} \in \theta$

3. $\langle\xi, \xi\rangle$ is a positive element of $Q$ for all $\xi \in \epsilon$ and $\langle\xi, \xi\rangle=0$ iff $\xi=0$.

(6) The group of unitary gauge transformations of 8

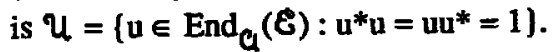

(7) A hermitian connection on is a linear map

$\nabla: \ell \rightarrow \& \otimes_{a} \Omega^{1}(a)$ such that

1. $\nabla(\xi a)=(\nabla \xi) a+\xi \otimes$ da for all $\xi \in \Theta, a \in Q$

2. $d\langle\xi, \eta\rangle=\langle\nabla \xi, \eta\rangle+\langle\xi, \nabla \eta>$ for all $\xi, \eta \in \Theta$.

We can extend $\nabla$ to a differentiation of $\Theta$-valued forms, $\nabla: \bullet \otimes_{a} \Omega^{*}(a) \rightarrow \& \otimes_{a} \Omega^{*}(a)$, by requiring

3. $\nabla(\xi \omega)=(\nabla \xi) \omega+\xi \otimes d \omega$ for all $\xi \in \varepsilon, \omega \in$ $\Omega^{*}(\mathcal{Q})$.
If we write $E$ in the form $B=e Q^{n}$ and $p$ is an $n \times n$ matrix of 1-forms, $\rho \in M_{n}(\alpha) \otimes_{a} \Omega^{1}(a)$, such that

ep $=\rho, \rho^{*}=-p$, then we obtain a Hermitian connection by putting $\nabla \xi=e d \xi+p \xi$, where $\xi \in Q^{n}$ and $e \xi=\xi$. Conversely, any Hermitian connection can be writtea in this form for some $\rho$. We will define the curvature of $\nabla$ to be $\theta=\nabla^{2} \in$ End $_{Q}\left(\varepsilon \otimes_{Q} \Omega^{*}(Q)\right)$. Acting $a n E$ alone, $\theta$ is an element of $\operatorname{End}_{Q}\left(\varepsilon, 8 \theta_{Q} \Omega^{2}(Q)\right)$.

There is a dual connection $\nabla^{*}: b^{*} \rightarrow \Omega^{1}(Q) \otimes_{Q} e^{*}$ defined by

$d(\eta, \xi)=\left(\nabla^{*} \eta, \xi\right)+(\eta, \nabla \xi)$ for all $\eta \in C^{*}, \xi \in G$.

For any element $u$ of $u$, we obtain a new Hermitian connection, the gauge transform of $\nabla$, by $\nabla^{\mathrm{a}}=\mathrm{u} \nabla \mathrm{u}^{-1}$. The curvature of $\nabla^{\mathbf{u}}$ is given by $\theta^{\mathfrak{u}}=u \theta^{-1}$.

(8) In order to proceed further, we will want a K-cycle on $a$, that is, a * representation of $a$ by bounded operators on a Hilbert space $g_{6}$ and a (possibly unbounded) self-adjoint operator $D$ on 96 such that

1. $[D, a]$ is a bounded operator for all $a \in Q$

2. $\left(1+D^{2}\right)^{-1}$ is a compact operator on $F$.

We will also assume a $\mathbb{Z}_{2}$ grading operator $\Gamma$ on If such that $\Gamma D+D \Gamma=0$ and $a \Gamma=\Gamma a$ for all $a \in Q$.

We will write $B(F 6)$ for the space of bounded operators on $H 6$ and $B\left(F, 26^{\prime}\right)$ for the space of bounded operators between two Hilbert spaces $\mathcal{H}$ and $\% 6$.

(9) If $G$ is a complex commutative algebra then any $K-$ cycle gives a metric $d$ on the character space $C$ by

$d(p, q)=\sup \{|p(f)-q(f)|: f \in Q,\|[D, f]\| \leq 1\}$.

(If $Q$ is a general $C^{*}$-algebra then the same definition gives a metric on the state space of $a$.)

(10) Define a map $\pi: \Omega *(Q) \rightarrow B(\%)$ by 
$\pi\left(a^{0} d a^{1} \ldots d^{k}\right)=a_{0} i\left[D, a_{1}\right] \ldots i\left[D, a_{k}\right]$.

This is an algebra homomorphism (but not a homomorphism of differential algebras). There are extensions of $\pi$, which we will also denote by $\pi$, to a (right) module homomorphism

$\pi: \& \otimes_{a} \Omega^{*}(a) \rightarrow B\left(F, \& \otimes_{a} F\right)$ and to a linear map

$\pi: \operatorname{End}_{Q}\left(\&, \& \otimes_{Q} \Omega^{*}(a)\right) \rightarrow B\left(\& \otimes_{Q}{ }^{H}\right)$.

(11) Given a hermitian connection $\nabla$, we obtain a selfadjoint operator $D_{\nabla}$ on the Hilbert space $\delta \otimes_{Q}$ अ by

$D_{\nabla}(\xi \otimes \eta)=\xi \otimes D \eta-i \pi(\nabla \xi) \eta$ for all $\xi \in \varepsilon, \eta \in \mathscr{F}$.

There is an action of $u$ on $\varepsilon \otimes_{Q}$ भ for which $D_{\nabla}$ is gauge covariant in the sense that $D_{\nabla} u=u D_{\nabla} u^{-1}$.

(12) We will define the spinor action as

$I_{\psi}=\left\langle\psi, D_{\nabla} \psi\right\rangle$ for $\psi \in \Theta \otimes_{a} H$.

In order to define the Yang-Mills action of a connection, we will need the notion of the Dixmier trace of a compact operator [Di]. Although we will only need this in a special case, where the Dixmier trace is effectively computable, we will give the general definition. For an infinite-dimensional Hilbert space $\mathfrak{F}$, define an ideal by

$$
\begin{gathered}
\mathscr{J}^{1+}(\mathscr{F})=\left\{T \in B(\mathscr{F}): T \text { is compact, } \sum_{i=0}^{N} \mu_{i}(T)=\right. \\
O(\log (N+1))\},
\end{gathered}
$$

where $\left\{\mu_{\mathrm{i}}\right\}$ are the eigenvalues of ITI. If $\omega$ is a mean on the space $1^{\infty}\left(\mathbb{Z}^{+}\right)$of bounded sequences on $\mathbb{Z}^{+}$, and $T$ is a positive element of $\mathfrak{b}^{1+}(\mathscr{W})$, define

$$
\operatorname{Tr} \omega(T)=\omega\left(\left\{\sum_{i=0}^{N} \mu_{i}(T) / \log (N+1)\right\}\right) .
$$

What is nontrivial is that if $\omega$ is chosen to be scale-invariant in an appropriate way then $\operatorname{Tr}_{\omega}$ is a linear functional and extends to a positive trace on $\mathfrak{J}^{1+}(\mathscr{H})$, the Dixmier trace. (If $\mathscr{H}$ is finite-dimensional then we will define $\operatorname{Tr}_{\omega}$ to be the usual trace.)

We will only need the Dixmier trace of pseudodifferential operators. Suppose that $\mathbf{Z}$ is $\mathbf{n}$-dimensional and that $\mathbf{P}$ is a pseudodifferential operator of order $-\mathrm{n}$ acting on sections of a vector bundle over $Z$. Then $P \in \mathfrak{S}^{1+}(\mathscr{H})$. Let $\sigma_{P}$ denote the principal symbol of $P$, a matrix-valued function on $T * Z$. Then regardless of the mean $\omega$ used to define the Dixmier trace, one has

$\operatorname{Tr}_{\omega}(P)=\left(n(2 \pi)^{n}\right)^{-1} \int_{S^{*} Z} \operatorname{trace}\left(\sigma_{P}\right) d \mu$

where $S * Z$ is the cosphere bundle of $Z$ (the subset of the cotangent bundle consisting of unit covectors) with the standard measure $d \mu[\mathrm{Co} 3]$. In this case one sees that the Dixmier trace is the integral of a local expression over $Z$.

\section{(13) Let us consider the Dixmier trace on}

$\mathfrak{L}^{1+}\left(B \otimes_{a} \mathscr{T C}\right)$. Suppose that $D$ is such that

$D^{-2 k} \in \mathfrak{S}^{1+}(\mathscr{G})$ for some integer $k$. (We will want to choose the smallest such $k$.) Then we will define the YangMills action to be $\mathrm{I}_{\nabla}=\operatorname{Tr}_{\omega}\left((\pi(\theta))^{2} \mathrm{D}_{\nabla}{ }^{-2 \mathrm{k}}\right)$. One can also define a topological action, for which we refer to [Co2].

(14) One can take the product of noncommutative spaces as follows. Let $\left(C_{1}, \mathscr{G}_{1}, D_{1}, \Gamma_{1}\right)$ and $\left(G_{2}, \mathscr{G}_{2}, D_{2}, \Gamma_{2}\right)$ be two noncommutative spaces with K-cycles. Form a new noncommutative space with $\mathrm{K}$-cycle by putting

$C=C_{1} \otimes C_{2}, \mathscr{C}=\mathscr{G}_{1} \otimes \mathscr{G}_{2}, D=D_{1} \otimes I+\Gamma_{1} \otimes D_{2}$ and $\Gamma=\Gamma_{1} \otimes \Gamma_{2}$.

The character space for $Q$ is $C=C_{1} \times C_{2}$. The space of differential forms $\Omega^{*}(a)$ maps to $\Omega^{*}\left(a_{1}\right) \otimes \Omega^{*}\left(a_{2}\right)$. Given vector bundles $E_{1}$ (associated to $Q_{1}$ ) and $E_{2}$ 
(associated to $\mathrm{C}_{2}$ ) with Hermitian metrics, there is a vector bundle $\bullet=\varepsilon_{1} \otimes \varepsilon_{2}$ associated to $G$ with Hermitian metric.

\section{IExamples}

\section{A. Commutative Geometry}

(1) Let $Z$ be an even-dimensional smooth compact spin manifold and take $G=C^{\infty}(Z) \otimes C$.

(2) $\mathrm{C}=\mathrm{Z}$, with $\mathrm{z} \in \mathrm{Z}$ corresponding to the homomorphism $f \rightarrow f(z)$.

(3) Take E to be a smooth finite-dimensional vector bundle over $Z$ and put $E=C^{\infty}(E)$, the space of smooth cross-sections of $E$. $E^{*}$ is the space of smooth crosssections of $\mathrm{E}^{*}$, the vector bundle whose transition functions are the inverse transpose of those of $E$.

(4) $\Omega^{\mathrm{k}}(\Theta)$ can be identified with the space of smooth functions $\mathrm{g}: \mathrm{Z}^{\mathrm{k}+1} \rightarrow \mathbb{C}$ such that

$g\left(z_{1}, \ldots, z_{i-1}, z_{i}, z_{i}, z_{i+2}, \ldots, z_{k+1}\right)=0$ for all $1 \leq i \leq k$. The left action of $Q$ is given by

$(f g)\left(z_{1}, \ldots, z_{k+1}\right)=f\left(z_{1}\right) g\left(z_{1}, \ldots, z_{k+1}\right)$ and the right action of $a$ is given by

$(g f)\left(z_{1}, \ldots, z_{k+1}\right)=g\left(z_{1}, \ldots, z_{k+1}\right) f\left(z_{k+1}\right)$.

Note that the right and left actions are not the same, even though $\alpha$ is commutative. The involution on $\Omega^{\mathbf{k}}(\alpha)$ is given by $g^{*}\left(z_{1}, \ldots, z_{k+1}\right)=(-1)^{k} g\left(z_{k+1}, \ldots, z_{1}\right)$.

The product of a $\mathbf{k}$-form $\mathrm{g}$ and a $\mathbf{k}$-form $\mathrm{g}$ ' is given by $\left(\mathrm{gg}^{\prime}\right)\left(\mathrm{z}_{1}, \ldots, \mathrm{z}_{\mathrm{k}+\mathrm{k}^{\prime}+1}\right)=$ $g\left(z_{1}, \ldots, z_{k+1}\right) g^{\prime}\left(z_{k+1}, \ldots, z_{k+k^{\prime}+1}\right)$
The differential on $\Omega^{*}(\mathcal{Q})$ is given by $(\mathrm{dg})\left(\mathrm{z}_{1}, \ldots, \mathrm{z}_{\mathrm{k}+2}\right)=$ $\sum_{i=1}^{k+2}(-1)^{i+1} g\left(z_{1}, \ldots, z_{i-1}, z_{i+1}, \ldots, z_{k+2}\right)$

(5) Any smooth hermitian inner product (.) on E gives a hermitian metric on $\varepsilon$ by sending tie pair $\xi_{1}, \xi_{2} \in \boldsymbol{C}$ to the function $\left\langle\xi_{1}, \xi_{2}\right\rangle \in Q$ defined by

$<\xi_{1}, \xi_{2}>(z)=\left(\xi_{1}(z), \xi_{2}(z)\right)$

(6) $น$ is the group of smooth unitary gauge transformations of $\mathrm{E}$.

(7) If $6=Q$ then a connection is specified by a skew element $\rho=\sum_{j} a_{j} d b_{j}$ of $\Omega^{l}(a)$. The curvature is $\theta=d p+p^{2} \in \Omega^{2}(Q)$. Note that the $p^{2}$ term does not vanish, unlike in the usual calculus of differential forms.

(8) Let $\mathrm{S}$ denote the vector bundle of spinors on $\mathrm{Z}$ and put $\mathscr{x}=\mathbf{L}^{2}(\mathbf{S})$, the Hilbert space of square-integrable sections of $S$. Let $D$ be the Dirac operator and let $\Gamma$ be the Hermitian chirality operator on $\mathscr{H}$.

(9) This reproduces the geodesic distance on $\mathrm{Z}$

(10) If we note by $d$ a the operator $\sum_{\mu} \gamma^{\mu} \partial_{\mu}$ a on 26 then $\pi\left(a^{0} \mathrm{da}^{1} \ldots \mathrm{da}^{\mathrm{k}}\right)=\mathrm{a}^{0} \mathrm{da} \mathrm{a}^{1} \ldots d \mathrm{a}^{\mathrm{k}}$

(11) If $G=Q$ then $D_{\nabla}=\not b-i \sum_{j} a_{j} d b_{j}$, an operator on $\mathrm{L}^{2}(\mathrm{~S})$, which is recognized to be the Dirac operator coupled to a U(1) gauge field.

(12) This gives the usual action for a spinor field in a U(1) background.

(13) If $Z$ is four-dimensional and $E$ is a $\mathbb{C}^{N}$ vector bundle over $\mathrm{Z}$ then one finds 
$I_{\nabla}=\left(8 \pi^{2}\right)^{-1} \int_{Z}\left[|F|^{2}+X^{2}\right] d v o l$

the sum of the usual Yang-Mills action and a Gaussian term which decouples. The topological action is proportionate to the usual topological charge $p_{1}(E)$.

(14) If $Z_{1}$ and $Z_{2}$ are ordinary spaces then $Q$ is the algebra corresponding to $Z=Z_{1} \times Z_{2}$. $T 6$ is the Hilbert space of square-integrable spinors on Z, D is the Dirac operator on $\mathrm{Z}$ and $\mathrm{\Gamma}$ is the Hermitian chirality operator for $Z$. If $E_{1}$ and $E_{2}$ arise from ordinary vector bundles $E_{1}$ (on $Z_{1}$ ) and $E_{2}$ (on $Z_{2}$ ) then $\varepsilon$ arises from the ordinary vector bundle $\pi_{1}^{*} E_{1} \otimes \pi_{2}{ }^{*} E_{2}$ on $Z$

\section{B. Two-Point Space}

(1) Take $\mathbb{Q}=\mathbb{C} \oplus \mathbb{C}$, with

$\left(\lambda_{1}, \lambda_{1}\right)+\left(\lambda_{2}, \lambda_{2}\right)=\left(\lambda_{1}+\lambda_{2}, \lambda_{1}^{\prime}+\lambda_{2}\right)$ and

$\left(\lambda_{1}, \lambda_{1}\right)\left(\lambda_{2}, \lambda_{2}\right)=\left(\lambda_{1} \lambda_{2}, \lambda^{\prime}{ }_{1}^{\prime} \lambda_{2}^{\prime}\right)$

(2) C is a two-point space, $C=\left\{p, p^{\prime}\right\}$, with

$p:(\lambda, \lambda) \rightarrow \lambda$ and $p^{\prime}:(\lambda, \lambda) \rightarrow \lambda:$

(3) Take $8=\mathbb{c}^{k} \oplus \mathbb{C}^{k^{\prime}}$, with the $Q$ action given by $\left(\xi, \xi^{\prime}\right)\left(\lambda, \lambda^{\prime}\right)=\left(\xi \lambda, \xi^{\prime} \lambda^{\prime}\right)$ for all $\left(\xi, \xi^{\prime}\right) \in \varepsilon$ and

$(\lambda, \lambda) \in Q$. If we assume that $k \leq k^{\prime}$ then we can write

$\varepsilon=\mathrm{e}^{\mathbf{k}^{\prime}}$ where $\mathrm{e}$ is a diagonal $\mathrm{k}^{\prime} \times \mathrm{k}^{\prime}$ matrix with entries consisting of $k(1,1)$ 's and $k^{\prime}-k(0,1)$ 's.

(4) $\Omega *(Q)$ has the same interpretation as in the previous example. In particular, $\Omega^{0}(a)=Q=\mathbb{C}^{2}$ and $\Omega^{1}(a)$ can be identified as $\boldsymbol{c}^{2}$, the values of $g$ at $\left(p, p^{\prime}\right)$ and $\left(p^{\prime}, p\right)$. The involution on $\Omega^{1}(Q)$ is given by $(r, s)^{*}=-\left(s^{*}, r^{*}\right)$ for all $(r, s) \in \mathbb{C}^{2}$. The differential $d: \Omega^{0}(a) \rightarrow \Omega^{1}(a)$ is given by $d\left(v, v^{\prime}\right)=\left(v^{\prime}-v, v-v^{\prime}\right)$.

(5) We can take $\left\langle\left(\xi_{1}, \xi_{1}\right),\left(\xi_{2}, \xi_{2}\right)\right\rangle=\left(\xi_{1}^{\uparrow} \xi_{2}, \xi_{1}{ }^{\uparrow} \xi_{2}\right) \in Q$

for all $\left(\xi_{1}, \xi_{1}\right),\left(\xi_{2}, \xi_{2}\right) \in \Theta$.

(6) $u$ is $U(k) \times U\left(k^{\prime}\right)$.

(7) If $\&=Q$ then a connection is specified by a skew element $\rho \in \Omega^{1}(Q)$, which we can write as

$p=\left(r, r^{*}\right) \in \mathbb{C}^{2}$

(8) Take $भ=\mathbb{C}^{\mathbf{N}} \oplus \mathbb{C}^{\mathbf{N}}$, with the action of $\mathrm{Q}$ given by $\left(\lambda, \lambda^{\prime}\right)\left(\eta, \eta^{\prime}\right)=\left(\lambda \eta, \lambda^{\prime} \eta^{\prime}\right)$

for all $\left(\lambda, \lambda^{\prime}\right) \in Q$ and $\left(\eta, \eta^{\prime}\right) \in \mathscr{H}$.

If $M$ is an $N \times N$ matrix, let $D$ be the operator $\left(\begin{array}{cc}0 & M^{+} \\ M & 0\end{array}\right)$ and let $\Gamma$ be the operator $\left(\begin{array}{cc}1 & 0 \\ 0 & -1\end{array}\right)$

(9) Let $L$ denote the square root of the inverse of the largest eigenvalue of $M^{\dagger} M$. Then the metric on $C$ is given by $d\left(p, p^{\prime}\right)=L$.

(10) If for $a=\left(\lambda, \lambda^{\prime}\right) \in Q$ we denote by $d a$ the operator

$i\left(\lambda-\lambda^{\prime}\right)\left(\begin{array}{cc}0 & -M^{\top} \\ M & 0\end{array}\right)$ then

$\pi\left(a^{0} d a^{1} \ldots d a^{k}\right)=a^{0} d a^{1} \ldots d a^{k}$. In particular, for a skew

1 -form $\rho=\sum_{j} a_{j}^{0}{ }_{j} a^{1}$, if we write $\rho$ in the form 
$\left(\phi^{*}-1, \phi-1\right) \in \mathbb{C}^{2}$ then $\pi(p)$ is the operator $\left(\begin{array}{cc}0 & i(\bar{\phi}-1) M^{2} \\ i(\phi-1) M & 0\end{array}\right)$ The image $\pi(\theta)$ of the curvature of $\rho$ is $\pi\left(d p+p^{2}\right)=\pi\left(\sum_{j} d a_{j}^{0} d a_{j}^{1}\right)+\pi\left(p^{2}\right)=$ $\sum_{j} \pi\left(\mathrm{da}^{0}{ }_{\mathrm{j}}\right) \pi\left(\mathrm{da}{ }_{\mathrm{j}}{ }_{\mathrm{j}}\right)+\pi(\rho)^{2}$, which turns out to be the operator $\left(1-|\phi|^{2}\right)\left(\begin{array}{cc}M^{\top} M & 0 \\ 0 & M M^{+}\end{array}\right)$.

(11) With the connection given by $\rho$ as above, $D_{\nabla}$ is the operator $\left(\begin{array}{ccc}0 & \overline{\phi M} \\ \phi M & 0\end{array}\right)$.

(12) The fermionic action is $L_{\psi}=\Psi^{\dagger} D_{\nabla} \Psi$ for $\psi \in \mathcal{H}$.

(13) The Yang-Mills action is

$I_{\nabla}=2\left(1-|\phi|^{2}\right)^{2} \operatorname{Tr}\left(M^{\dagger} M\right)^{2}$. When $\varepsilon=a$, the topological action vanishes. (More generally, the topological action will be proportionate to $\mathbf{k}-\mathrm{k}^{\mathbf{k}}$.)

(14) We can take the product of noncommutative geomerries corresponding to two two-point spaces to get a noncommutative geometry for a four-point space, in a straightforward way.

\section{Glashow-Weinberg-Salam Model.}

Let $Z$ be a closed Riemannian spin 4-manifold. (We will make the transition later to a Lorentzian spacetime). Let $S$ denote the spinor bundle and let $\gamma \in B\left(L^{2}(S)\right)$ denote the Hermitian chirality operator. Let $N_{G}$ be the number of generations and let $M$ be an $N_{G} \times N_{G}$ matrix, the mass matrix.

In order to build our models, the general strategy will be to think of the algebra $Q$ and the vector bundle 8 logether as specifying the gauge group, and the action of $Q$ on the Hilbert space 26 as specifying the fermionic representation of the gauge group. For example, suppose that we take $Q$ to be $C^{\infty}(Z)$ and 6 to be $\mathbb{C}^{N} \otimes C^{\infty}(Z)$. Suppose that the action of $f \in Q$ on $E$ is given by $I_{N} \otimes f$, that is,

$(v \otimes g) f=v \otimes g f$. Then the unitary gauge group is $\operatorname{Map}(\mathrm{Z}, \mathrm{U}(\mathrm{N}))$. Suppose that $\mathscr{H}$ is $\mathrm{L}^{2}(\mathrm{~S}) \otimes \mathbb{C}^{\mathrm{M}}$ and that the action of $f \in Q$ on 96 is given by $f \otimes I_{M}$, that is,

$f(\eta \otimes w)=f \eta \otimes w$. Then the space of fermions is

$\epsilon \otimes a r=\mathbb{C}^{N} \otimes L^{2}(S) \otimes \mathbb{C}^{M}$. In other words, we obtain $M$ distinct fermions that are in the fundamental representation of $\mathbf{U}(\mathrm{N})$.

To write the GWS model, we will first take the structure group to be $U(1) \times U(2)$ and later restrict to $U(1) \times S U(2)$. We have seen in example $A$ of the previous section that when one specializes noncommutative gauge theory to the usual commutative case, one recovers the standard formalism of gauge fields and Yang-Mills actions. In example $B$ we saw that when one does gauge theory on a two-point space, with an off-diagonal Dirac operator, one obtains a variable $\phi$, which we can interpret as a Higgs field. The Yang-Mills action in example B was exactly a symmetry-breaking Higgs potential. In oxder to write the GWS model, we will simply take the product of the noncommutative spaces of examples A and B. Let us write our two factor spaces as noncommutative spaces with $\mathrm{K}$ cycles, $\left(Q_{1}, \mathscr{G}_{1}, D_{1}, \Gamma_{1}\right)$ and $\left(G_{2}, \mathscr{F}_{2}, D_{2}, \Gamma_{2}\right)$.

$\left(Q_{1}, F_{1}, D_{1}, \Gamma_{1}\right)$ will correspond to the usual Riemannian geometry on $Z$ :

$a_{1}=C^{\infty}(Z), H_{1}=L^{2}(S), D_{1}=\square$ and $\Gamma_{1}=\gamma$. 
For $\left(G_{2}, H_{2}, D_{2}, \Gamma_{2}\right)$, we will take

$a_{2}=\mathbb{C} \oplus \mathbb{C}, \mathscr{F}_{2}=(\mathbb{C} \oplus \mathbb{C}) \otimes \mathbb{C}^{N_{G}}, D_{2}=\left(\begin{array}{cc}0 & M^{\uparrow} \\ M & 0\end{array}\right)$ and $\Gamma_{2}=\left(\begin{array}{rr}1 & 0 \\ 0 & -1\end{array}\right)$

If we write an element of $\mathscr{F}_{2}$ in the form $\left(\eta, \eta^{\prime}\right)$ with

$\eta, \eta^{\prime} \in \mathbb{C}^{N_{G}}$ then the action of $\left(\lambda, \lambda^{\prime}\right) \in C_{2}$ on

$\left(\eta, \eta^{\prime}\right) \in \mathscr{H}_{2}$ will be taken to be

$\left(\lambda, \lambda^{\prime}\right)\left(\eta, \eta^{\prime}\right)=\left(\lambda \eta, \lambda^{\prime} \eta^{\prime}\right)$. For the product geometry

(A, $36, D, \Gamma)$, we have

$G=C^{\infty}(Z) \oplus C^{\infty}(Z), \mathscr{C}=L^{2}(S) \otimes \mathbb{C}^{2} \otimes \mathbb{C}^{N_{G}}$,

$D=\not B \otimes I_{2} \otimes I_{N_{G}}+\gamma \otimes\left(\begin{array}{cc}0 & M^{\uparrow} \\ M & 0\end{array}\right)$ and

$\Gamma=\gamma \otimes\left(\begin{array}{cc}1 & 0 \\ 0 & -1\end{array}\right) \otimes I_{N_{G}}$

The character space $C_{1}$ of $\left(C_{1}, G_{1}, D_{1}, \Gamma_{1}\right)$ is $Z$ and the character space $C_{2}$ of $\left(C_{2},{ }^{*} C_{2}, D_{2}, \Gamma_{2}\right)$ is a two-point space. Thus the character space $\mathrm{C}$ of the product geometry is the disjoint union of two copies of $Z$, say $Z$ and $Z$. Let us denote the geodesic distance on $Z$ by $d_{Z}$. Let $L$ denote the square root of the inverse of the largest eigenvalue of $M^{\uparrow} M$. One can show that the distance $d$ on $C$ satisfies

$d(p, q)=d_{Z}(p, q)$ for $p, q \in Z$,

$d\left(p^{\prime}, q^{\prime}\right)=d_{Z^{\prime}}\left(p^{\prime}, q^{\prime}\right)$ for $p^{\prime}, q^{\prime} \in Z^{\prime}$,

$\left(d_{Z}\left(p, p^{\prime}\right)^{2}+L^{2}\right)^{1 / 2} \leq d\left(p, p^{\prime}\right) \leq d z\left(p, p^{\prime}\right)+L$

for $p \in Z, p^{\prime} \in Z^{\prime}$.
Thus the picture is of two copies of $Z$ separated by a distance $L$. (As one loses information in passing to the character space, this picture should not be taken too literally.)

For the vector bundles, let us take $\varepsilon_{1}=a_{1}$ and

$\mathbb{\varepsilon}_{2}=\mathbb{C} \oplus \mathbb{C} \oplus \mathbb{C}$, with the action of $\left(\lambda, \lambda^{\prime}\right) \in \mathfrak{C}_{2}$ on $\left(\xi_{1}, \xi_{2}, \xi_{3}\right) \in \epsilon_{2}$ given by

$\left(\xi_{1}, \xi_{2}, \xi_{3}\right)\left(\lambda, \lambda^{\prime}\right)=\left(\xi_{1} \lambda, \xi_{2} \lambda^{\prime}, \xi_{3} \lambda^{\prime}\right)$. Then the vector bundle $\mathcal{E}$ is $\mathrm{C}^{\infty}(\mathrm{Z}) \oplus \mathrm{C}^{\infty}(\mathrm{Z}) \oplus \mathrm{C}^{\infty}(\mathrm{Z})$. This can be written more succinctly as $E=\mathrm{ea}^{2}$, with $\mathrm{e}=\left(\begin{array}{cc}(1,1) & 0 \\ 0 & (0,1)\end{array}\right)$ . The unitary gauge group of $E$ is $\operatorname{Map}(Z, U(1) \times U(2))$. In terms of the character space, $\&$ can be thought of as a $\mathbb{C}^{1}$ bundle over $\mathrm{Z}$ and a $\mathbb{C}^{2}$ bundle over $\mathrm{Z}$. The fermion space is given by $\& \otimes a \mathfrak{G}=L^{2}(S) \otimes \mathbb{C}^{3} \otimes \mathbb{C}^{N_{G}}$.

We can write elements of $\epsilon \otimes_{Q} F$ in the form $\left(\begin{array}{l}e_{R} \\ e_{L} \\ v_{L}\end{array}\right)$, where $e_{R}, e_{L}, v_{L} \in L^{2}(S) \otimes \mathbb{C}^{N_{G}}$. In this way, $e_{R}$ is in the fundamental representation of $U(1)$ and $\left(e_{L}, v_{L}\right)$ is in the fundamental representation of $U(2)$.

Let us write a Hermitian connection $\nabla$ on $\varepsilon=\mathrm{eq}^{2}$ in the form $\nabla \xi=e d \xi+\rho \xi$,

where $\xi \in a^{2}, e \xi=\xi, \rho \in M_{2}(a) \otimes_{a} \Omega^{1}(a), e p=p$.

The matrix $\rho$ is a $2 \times 2$ matrix of one forms, the components of which can be written as

$$
\rho_{k l}=\sum_{j} a_{k l j} d b_{k l j}, a_{k l j}=\left(f_{k l j}, f^{\prime}{ }_{k l j}\right), b_{k l j}=\left(g_{k l j}, g_{k l j}^{\prime}\right),
$$

$$
1 \leq \mathrm{k}, 1 \leq 2 .
$$

The condition e $\rho=\rho$ becomes $\mathrm{f}^{\prime}{ }_{21 \mathrm{j}}=\mathrm{f}^{\prime}{ }_{22 \mathrm{j}}=0$. 
Let us fix $k$ and $l$ for the moment. Each $a_{k l j}$ and $b_{k l j}$ acts on 16 as a $2 \times 2$ diagonal matrix. Then

$\pi\left(\rho_{k l}\right)=\sum_{j} a_{k l j} i\left[D, b_{k l j}\right]$ is a $2 \times 2$ matrix with components

$\pi\left(\rho_{k l}\right)_{11}=\sum_{j} f_{k l j} d g_{k l j} \otimes I_{N_{G}}$

$\pi\left(\rho_{\mathrm{kl}}\right)_{12}=\sum_{\mathrm{j}}-\mathrm{if}_{\mathrm{klj}}\left(\mathrm{g}_{\mathrm{klj}} \mathrm{g}_{\mathrm{klj}}^{\prime}\right) \gamma \otimes \mathrm{M}^{\dagger}$

$\pi\left(\rho_{k l}\right)_{2 l}=\sum_{j}$ if $^{\prime} k_{j j}\left(g_{k l j}-g_{k l j}^{\prime}\right) \gamma \otimes M$

$\pi\left(\rho_{\mathrm{kl}}\right)_{22}=\sum_{\mathbf{j}} \mathbf{f}_{\mathbf{k l j}^{\prime}} \boldsymbol{d g}_{\mathbf{k l j}^{\prime}} \otimes \mathrm{I}_{\mathrm{N}_{\mathrm{G}}}$

(We are using the notation that for a function $\mathrm{g}$,

$\left.d g=i[\not, g]=\sum_{\mu} \gamma^{\mu} \partial_{\mu} g.\right)$

We have that the $4 \times 4$ matrix $\pi(p)$, an operator on

$E \otimes_{Q} Y G$, has vanishing third row. By its skewHermiticity, it also has vanishing third column. Thus we can write $\pi(\rho)$ as a $3 \times 3$ matrix. One finds that it can be written in the form

$$
\pi(\rho)=\left(\begin{array}{ccc}
A \otimes I_{N_{G}} & i\left(\bar{\phi}_{1}-1\right) \gamma \otimes M^{\dagger} & -\bar{i}_{2} \gamma \otimes M^{\dagger} \\
i\left(\phi_{1}-1\right) \gamma \otimes M & A^{\prime} \otimes I_{N_{G}} & -W^{*} \otimes I_{N_{G}} \\
i \phi_{2} \gamma \otimes M & W \otimes I_{N_{G}} & Z \otimes I_{N_{G}}
\end{array}\right) .
$$

Here $A=\sum_{j} f_{11 j} d g_{11 j}, A^{\prime}=\sum_{j} f_{11 j} d g^{\prime}{ }_{11 j}$
$W=\sum_{j} f^{\prime}{ }_{21 j} d^{d g^{\prime}}{ }_{21 j}, Z=\sum_{j} f^{\prime}{ }_{22 j} d_{g^{\prime}}{ }_{22 j}{ }^{\prime}$

$\phi_{1}-1=\sum_{j} f^{\prime}{ }_{11 j}\left(g_{11 j}-g^{\prime}{ }_{11 j}\right)$ and

$\phi_{2}=\sum_{j} f^{\prime}{ }_{21 j}\left(g_{21} j^{-g_{21 j}^{\prime}}\right)$. In terms of their behaviour under gauge transformations, $A$ is an ordinary $U(1)$ gauge field, $\left(\begin{array}{cc}A^{\prime} & -W^{*} \\ W & Z\end{array}\right)$ is a $U(2)$ gauge field and

$\Phi=\left(\begin{array}{l}\phi_{1} \\ \phi_{2}\end{array}\right)$ is a Higgs doublet with covariant derivative

$D \Phi=d \Phi+\left(\begin{array}{cc}A^{\prime}-A & -W^{*} \\ W & Z-A\end{array}\right) \Phi$

(In terms of the intuiive picture of the geometry given by the character sface, the $U(1)$ gauge field is locally given by a differential form on $\mathrm{Z}$ and the $\mathrm{U}(2)$ gange field is locally given by differential forms on $Z$. The Higgs fields involve the differences of functions on $Z$ and $Z$, and so can be thought of as differential forms where the differential is replaced by a difference operator.)

The curvature $\theta=\nabla^{2}: \varepsilon \rightarrow \varepsilon \otimes_{a} \Omega^{2}(a)$ of the connection is $\theta=e$ de de $+e d \rho+\rho^{2}$. Its image $\pi(\theta)$, a self-adjoint operator on $\epsilon \otimes_{a} \mathfrak{H}$, can be written as a $3 x$ 3 matrix $T$ of operators. Tne only subtlety in computing $T$ comes from the fact that $T$ is locally an operator with values in the even part of the Clifford algebra. It does not consist only of Clifford algebra elements of degree two, as one might expect for a curvature. For example, $T_{11}$ has a $\operatorname{term} A^{2} \otimes I_{N_{G}}$. Because $A$ is an operator of the form 
$A=\sum_{\mu} A_{\mu} \gamma^{\mu}$, instead of vanishing, $A^{2}$ is actually a scalar field $\sum_{\mu} \mathrm{A}_{\mu}{ }^{2}$ of degree zero. In general, the product of two of our operator 1-forms can be decomposed in the Clifford algebra into the sum of a 2-form and a 0-form. Keeping this in mind, when decomposed into its various degrees we can write $T$ as

$T_{11}=F_{A} \otimes I_{N_{G}}+X \otimes I_{N_{G}}-\left(|\Phi|^{2}-1\right) \otimes M^{\uparrow} M$

$$
\begin{aligned}
& \left(\begin{array}{l}
T_{21} \\
T_{31}
\end{array}\right)=i(D \Phi) \gamma \otimes M
\end{aligned}
$$

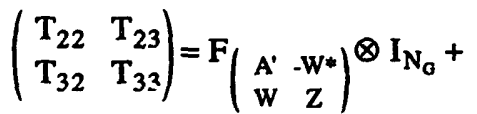

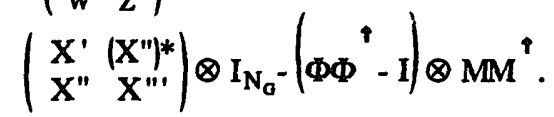

Here $X, X^{\prime}, X^{\prime \prime}$ and $X^{\prime \prime \prime}$ are new scalar fields.

In order to obtain a $U(1) \times S U(2)$ gauge theory, we must relate the $U(1)$ gauge field to the $U(1)$ part of the $U(2)$ gauge field. In terms of the hypercharge generator $Y$, we want $A=-2 Y$ and $A^{\prime}+Z=-2 Y$. Thus we will require $\mathrm{A}=\mathrm{A}^{\prime}+\mathrm{Z}$.

The Yang-Mills action $\left.\mathrm{I}_{\nabla}=\operatorname{Tr}_{\omega}\left((\pi(\theta))^{2} \mathrm{D}_{\nabla}{ }^{-4}\right)\right)$ is the sum of three terms:

$$
\begin{aligned}
S_{1}= & \left(8 \pi^{2}\right)^{-1} \int_{Z}\left[N_{G} \mid F_{A^{\prime}}{ }^{2}+\right. \\
& N_{G}\left(X-N_{G}^{-1}\left(\operatorname{Tr} M^{\dagger} M\right)\left(|\Phi|^{2}-1\right)\right)^{2}+ \\
& \left.\left(|\Phi|^{2}-1\right)^{2} \operatorname{Tr}\left(M^{\dagger} M-N_{G}{ }^{-1}\left(\operatorname{Tr} M^{\uparrow} M\right) I_{N_{G}}\right)^{2}\right] d v o l
\end{aligned}
$$

$S_{2}=2\left(8 \pi^{2}\right)^{-1} \operatorname{Tr} M^{\uparrow} M \int_{Z} D \Phi^{\uparrow} D \Phi d v o l$

$$
\begin{aligned}
S_{3}= & \left(8 \pi^{2}\right)^{-1} \int_{Z}\left[N_{G}\left|F_{U(2)}\right|^{2}+\right. \\
& N_{G} \operatorname{Tr}\left(X_{U(2)}-N_{G}{ }^{-1}\left(\operatorname{Tr} M^{\dagger} M\right)\left(\Phi \Phi^{\dagger}-I\right)\right)^{2}+ \\
& \left.\left(\left(|\Phi|^{2}-1\right)^{2}+1\right) \operatorname{Tr}\left(M^{\dagger} M-N_{G}{ }^{-1}\left(\operatorname{Tr} M^{\dagger} M\right) I_{N_{G}}\right)^{2}\right] \\
& \text { dvol. }
\end{aligned}
$$

Because the auxiliary fields $X$ and $X_{U(2)}$ appear quadratically and algebraically, one can immediately minimize over them. If there is more than one generation, and not all of the electron-like fermions in the various generations have the same mass, then we obtain a symmetry-breaking Higgs potential. (If there is one generation then $M^{\dagger} M-N_{G}^{-1}\left(\operatorname{Tr} M^{\dagger} M\right) I_{N_{G}}$ vanishes and we do not have a Higgs potential). We will discuss the question of relations among the coupling constants in section VI.

The fermionic action is

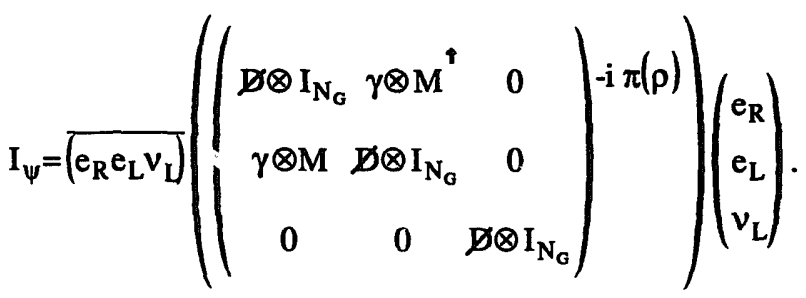

In order to write an action for a Lorentzian spacetime, we will do the obvious changes for the bosons, and require that the fermion field $\psi \in E \otimes_{a} \mathscr{F}$ satisfy $\Gamma \psi=\psi$. In this way we obtain the GWS model [Gl,We1,Sa].

\section{The Peccei-Quinn Model.}

We now want to add the quarks to the GWS model. This will lead to the Peccei-Quinn model. The idea is that 
the triplets $\left(\begin{array}{l}e_{R} \\ e_{L} \\ v_{L}\end{array}\right)$ and $\left(\begin{array}{l}d_{R} \\ d_{L} \\ u_{L}\end{array}\right)$ differ in their $U(1) \times S U(2)$ properties by a total hypercharge. The $u_{R}$ quark is left over. This suggests having an algebra $Q$ with vector bundle $E$, with gauge group $U(1) \times U(1) \times U(2)$. The first $U(1)$ will act on $u_{R}$, the second $U(1)$ will act on $e_{R}$ and $d_{R}$, and the $U(2)$ will act on $\left(e_{L}, v_{L}\right)$ and $\left(d_{L}, u_{L}\right)$. We also have to add the color degrees of freedom to the quarks. If we can do this then the gluons are automatically generated in our model. In order to provide the color we will add a new algebra of which acts on the Hilbert space, and a vector bundle $\mathfrak{F}$ associated to $\mathcal{B}$. If the gauge group of $\mathfrak{F}$ were taken to just be $U(3)$, then all of the physical fermions would have to have color. Thus we will take $\mathbb{B}$ and $\mathcal{F}$ so that the gauge group of $\mathcal{F}$ is $U(1) \times U(3)$. The U(1) will act on the leptons and the $U(3)$ will act on the quarks. The total gauge group is now $U(1) \times U(1) \times U(2) \times U(1) \times$ $U(3)$. When the various $U(1)$ factors are related, the final gange group will be $U(1) \times S U(2) \times S U(3)$. There will be no Higgs fields associated to the gauge group of $\Im$, and so the SU(3) gauge group will be unbroken.

Let us first describe the geometry of our model over a point in $Z$. $Q$ will be the algebra of a three-point space:

$\boldsymbol{a}=\mathbb{C} \oplus \mathbb{C} \oplus \mathbb{C}$. Let $\mathbb{B}=\mathbb{C} \oplus \mathbb{C}$ be an auxiliary algebra, which one can think of as taking the place of $\mathbb{C}$ as a ground ring. Take $\mathscr{H}$ to be $\left(\mathbb{C} \oplus \mathbb{C}^{2} \oplus \mathbb{C}^{2}\right) \oplus \mathbb{C}^{N} \mathbf{G}$. (One can think that the first factor in $\mathcal{H}$ corresponds to $u_{R}$, the second factor corresponds to $e_{R}$ and $d_{R}$, and the third factor corresponds to the four left-handed fermions.) The (left) action of $\left(f, f^{\prime}, f^{\prime \prime}\right) \in \mathcal{Q}$ on $\mathscr{H}$ is given by $\left(f I_{1} \oplus f^{\prime} I_{2} \oplus f^{\prime \prime} I_{2}\right) \otimes I_{N_{G}}$, and the (right) action of (g,g') $\in \mathbb{B}$ on $\mathcal{H}$ is given by $\left(\mathrm{g}^{\prime} \mathrm{I}_{1} \oplus\left(\begin{array}{ll}\mathrm{g} & 0 \\ 0 & \mathrm{~g}^{\prime}\end{array}\right) \oplus\left(\begin{array}{ll}\mathrm{g} & 0 \\ 0 & \mathrm{~g}^{\prime}\end{array}\right)\right) \otimes \mathrm{I}_{\mathrm{N}_{\mathrm{G}}}$. The operator $\mathrm{D}$ will be
$D=\left(\begin{array}{ccc}0 & 0 & M_{2}^{\uparrow} \\ 0 & 0 & M_{3}^{\uparrow} \\ M_{2} & M_{3} & 0\end{array}\right)$, where $M_{2}=\left(\begin{array}{c}0 \\ M_{v}\end{array}\right)$ is a $2 N_{G} \times N_{G}$ matrix and $M_{3}=\left(\begin{array}{cc}M_{e} & 0 \\ 0 & M_{d}\end{array}\right)$ is a $2 N_{G} \times 2 N_{G}$ matrix. Note that requiring that $D$ commutes with the action of $B$ gives $M_{2}$ and $M_{3}$ their special forms. The $Z_{2}$-grading operator will be $\Gamma=\left(I_{1} \oplus I_{2} \oplus\left(-I_{2}\right)\right) \otimes I_{N_{G}}$.

Now let us give the full model. Let $a$ be

$C^{\infty}(Z) \oplus C^{\infty}(Z) \oplus C^{\infty}(Z)$, 33 be $C^{\infty}(Z) \oplus C^{\infty}(Z)$ and

96 be $L^{2}(S) \otimes\left(\mathbb{C} \oplus \mathbb{C}^{2} \oplus \mathbb{C}^{2}\right) \otimes \mathbb{C}^{N_{G}}$. The (left) action of $\left(f, f^{\prime}, f "\right) \in \mathcal{Q}$ on $x$ is given by

$\left(f I_{1} \oplus f^{\prime} I_{2} \oplus f^{\prime \prime} I_{2}\right) \otimes I_{N_{G}}$, and the (right) action of

$\left(\mathrm{g}, \mathrm{g}^{\prime}\right) \in \mathbb{B}$ on $\mathrm{H} 6$ is given by

$\left(g^{\prime} I_{1} \oplus\left(\begin{array}{cc}g & 0 \\ 0 & g^{\prime}\end{array}\right) \oplus\left(\begin{array}{cc}g & 0 \\ 0 & g^{\prime}\end{array}\right)\right) \otimes I_{N_{G^{*}}}$ The Dirac operator is

$D=D \otimes\left(I_{1} \oplus I_{2} \oplus I_{2}\right) \otimes I_{N_{G}}+\gamma \otimes\left(\begin{array}{ccc}0 & 0 & M_{2} \\ 0 & 0 & M_{3}{ }^{\imath} \\ M_{2} & M_{3} & 0\end{array}\right)$

where $M_{2}=\left(\begin{array}{c}0 \\ M_{u}\end{array}\right)$ is a $2 N_{G} \times N_{G}$ matrix and

$M_{3}=\left(\begin{array}{cc}M_{e} & 0 \\ 0 & M_{d}\end{array}\right)$ is a $2 N_{G} \times 2 N_{G}$ matrix. The $\mathbb{Z}_{2^{-}}$ grading operator is $\Gamma=\gamma \otimes\left(I_{1} \oplus I_{2} \oplus\left(-I_{2}\right)\right) \otimes I_{N_{G}}$. 
Take $\mathcal{E}=C^{\infty}(Z) \oplus C^{\infty}(Z) \oplus C^{\infty}(Z) \oplus C^{\infty}(Z)=e a^{2}$, with $e=\left(\begin{array}{cc}(1,1,1) & 0 \\ 0 & (0,0,1)\end{array}\right)$, and

$\mathcal{F}=C^{\infty}(Z) \oplus C^{\infty}(Z) \oplus C^{\infty}(Z) \oplus C^{\infty}(Z)=\mathbb{B}^{3} f$, with

$f=\left(\begin{array}{ccc}(1,1) & 0 & 0 \\ 0 & (0,1) & 0 \\ 0 & 0 & (0,1)\end{array}\right)$. The gauge group of $\&$ is $\operatorname{Map}(Z, U(1) \times U(1) \times U(2))$ and the gauge group of $\mathfrak{F}$ is $\operatorname{Map}(Z, U(1) \times U(3))$.

An element of the physical Hilbert space

$\mathcal{P}=\mathcal{E} \otimes_{a} \mathfrak{H} \otimes_{B B} \mathcal{F}$ can be written as

$$
\begin{gathered}
\left(u_{R}, e_{R}, d_{R}, e_{L}, d_{L}, v_{L}, u_{L}\right) \in L^{2}(S) \otimes\left(\mathbb{C}^{3} \oplus\left(\mathbb{C}^{1} \oplus \mathbb{C}^{3}\right) \oplus\right. \\
\left.\left(\mathbb{C}^{1} \oplus \mathbb{C}^{3}\right) \oplus\left(\mathbb{C}^{1} \oplus \mathbb{C}^{3}\right)\right) \otimes \mathbb{C}^{N_{G}} .
\end{gathered}
$$

A connection on $\mathcal{F}$ will provide a $U(1)$ gauge field and a $\mathrm{U}(3)$ gauge field, and no Higgs fields. If a connection on $\delta$ is given by the matrix $\rho$ then one computes that $\pi(\rho)$, an operator on $E \otimes_{a} \mathcal{H}$, is a $4 \times 4$ matrix of operators

$\pi(\rho)=\left(\begin{array}{cccc}A \otimes I_{N_{0}} & 0 & i\left(\bar{\phi}_{2}-1\right) \gamma \otimes M_{2}, & i \bar{\phi}_{4} \gamma \otimes M_{2}{ }^{\prime} \\ 0 & A^{\prime} \otimes I_{N_{0}} & i\left(\bar{\phi}_{3}-1\right) \gamma \otimes M_{3}{ }^{\prime} & i \bar{\phi}_{5} \gamma \otimes M_{3}{ }^{\prime} \\ i\left(\phi_{2}-1\right) \gamma \otimes M_{2} & i\left(\phi_{3}-1\right) \gamma \otimes M_{3} & A^{\prime \prime} \otimes I_{N_{G}} & -W^{*} \otimes I_{N_{G}} \\ i \phi_{4} \gamma \otimes M_{2} & i \phi_{5} \gamma \otimes M_{3} & W \otimes I_{N_{G}} & Z \otimes I_{N_{G}}\end{array}\right)$.

(We are thinking of an element of $\& \otimes_{a} \mathscr{H}$ as $u_{R} \oplus\left(e_{R}, d_{R}\right) \oplus\left(e_{L}, d_{L}\right) \oplus\left(v_{L}, u_{L}\right)$. $) A$ and $A^{\prime}$ are $U(1)$ gauge fields and $\left(\begin{array}{cc}A^{\prime \prime} & -W^{*} \\ W & Z\end{array}\right)$ is a $U(2)$ gauge field. There are two Higgs doublets, $\Phi_{1}=\left(\begin{array}{l}\phi_{2} \\ \phi_{4}\end{array}\right)$ and $\Phi_{2}=\left(\begin{array}{l}\phi_{3} \\ \phi_{5}\end{array}\right)$, with covariant derivatives $\mathrm{D} \Phi_{1}=\mathrm{d} \Phi_{1}+\left(\begin{array}{cc}\mathrm{A}^{\prime \prime}-\mathrm{A}-\mathrm{W}^{*} \\ \mathrm{~W} & \mathrm{Z}-\mathrm{A}\end{array}\right) \Phi_{1}$, $\mathrm{D} \Phi_{2}=\mathrm{d} \Phi_{2}+\left(\begin{array}{cc}A^{\prime \prime}-A^{\prime} & -\mathrm{W}^{*} \\ \mathrm{~W} & \mathrm{Z}-\mathrm{A}^{\prime}\end{array}\right) \Phi_{2}$

The image $\pi(\theta)$ of the $\Theta$-curvature, a self-adjoint operator on $\& \otimes_{a} \mathcal{H}$, can be written as a $4 \times 4$ matrix $T$ of operators, with

$$
\begin{aligned}
& T_{11}=F_{A} \otimes I_{N_{G}}+X \otimes I_{N_{G}}-\left(\left|\Phi_{1}\right|^{2}-1\right) \otimes M_{2}^{\top} M_{2} \\
& \mathrm{~T}_{22}=\mathrm{F}_{\mathrm{A}^{\prime}} \otimes \mathrm{I}_{\mathrm{N}_{\mathrm{G}}}+\mathrm{X}^{\prime} \otimes \mathrm{I}_{\mathrm{N}_{\mathrm{G}}}-\left(\left|\Phi_{2}\right|^{2}-1\right) \otimes \mathrm{M}_{3}^{\dagger} \mathrm{M}_{3}
\end{aligned}
$$

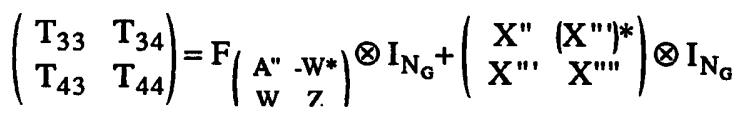

$$
-\left(\Phi_{1} \Phi_{1}^{\top}-\mathrm{I}\right) \otimes M_{2} M_{2}^{\top}-\left(\Phi_{2} \Phi_{2}^{\top}-\mathrm{I}\right) \otimes M_{3} M_{3}^{\top}
$$

$\mathrm{T}_{21}=\left(\phi_{1}-\Phi_{2}^{\uparrow} \Phi_{1}\right) \otimes \mathrm{M}_{3}^{\dagger} \mathrm{M}_{2}$

$\left(\begin{array}{l}T_{31} \\ T_{41}\end{array}\right)=i\left(D \Phi_{1}\right) \gamma \otimes M_{2}$

$\left(\begin{array}{l}T_{32} \\ T_{42}\end{array}\right)=i\left(D \Phi_{2}\right) \gamma \otimes M_{3}$.

Here $X, X^{\prime}, X^{\prime \prime}, X^{\prime \prime \prime}, X^{\prime \prime \prime}$ and $\phi_{1}$ are new auxiliary scalar fields.

We now have a $U(1) \times U(1) \times U(2) \times U(1) \times U(3)$ gauge theory. In order to reduce this to $U(1) \times S U(2) \times$ $S U(3)$, let us put that the first $U(1)$ part $A$ of the connection on $E$ vanishes, the second $U(1)$ part $A^{\prime}$ of the connection on $E$ is - $2 Y$, the trace of the $U(2)$ part of the connection on $E$ is $-2 Y$, the $U(1)$ part of the connection on $\mathcal{F}$ vanishes and the trace of the $U(3)$ part of the connection on $\mathcal{F}$ is $4 \mathrm{Y}$.

Upon computing the action as in section III, we obtain quartic potentials for $\Phi_{1}$ and $\Phi_{2}$. There is also a mixed 
term coming from $\left(\begin{array}{l}T_{33} T_{34} \\ T_{43} T_{44}\end{array}\right)$ which is proportionate to $\left|\Phi_{1}{ }^{\top} \Phi_{2}\right|^{2}$. Putting everything together, we obtain the Peccei-Quinn model [PQ]. This model has an axion [We2,Wi] which seems to be ruled out experimentally [EG].

Let us note that because the first $U$ (1) gauge field arising from $\Theta$ and the U(1) part of the gauge field arising from $\Im$ both vanish, we could have taken the algebras to be $\boldsymbol{C}=\boldsymbol{C}$ $\oplus \mathrm{C}^{\infty}(\mathrm{Z}) \oplus \mathrm{C}^{\infty}(\mathrm{Z})$ and $\mathbb{B}=\mathbb{C} \oplus \mathrm{C}^{\infty}(\mathrm{Z})$. The only effect that this would have would be to enforce the vanishing from the beginning.

\section{The Standard Model.}

In the standard model the Yukawa term which gives mass to the up quark involves not the Higgs doublet $\Phi$, but the field $\Phi^{\prime}=\mathrm{J} \bar{\Phi}$, where $J=\left(\begin{array}{cc}0 & 1 \\ -1 & 0\end{array}\right) \Phi^{\prime}$ has the same isospin as $\Phi$, but opposite hypercharge. One can use $\Phi^{\prime}$ to form a gauge-invariant action because the fundamental representation of SU(2) is unitarily equivalent to its complex conjugate. That is,

\section{$(*) \quad \mathbf{J} \overline{\mathbf{g}}=\mathbf{g} \mathbf{J}$}

for all $g \in S U(2)$. Our choice of the algebra $a$ for the standard model is based on the observation that $\left(^{*}\right)$ defines a subalgebra of $M_{2}(\mathbb{C})$, namely the quatemion algebra $\mathbb{H}$.

Let us first consider an example with the algebra $Q=\mathrm{H}$ and a vector bundle $\varepsilon=\mathbb{H}$, with $Q$ acting on $\varepsilon$ by right multiplication. Then End $(\varepsilon)$ equals $H$, acting on $\&$ by left multiplication. The unitary gauge group is the group of unitary quaternions, nimely SU(2).

This suggests taking the algebra $Q$ for the standard model to be $C^{\circ}(Z) \otimes(\mathbb{C} \oplus \mathbb{H})$. We will add the glunons later. We will first work with complex vector spaces and describe directly the full model. We will later show how to describe the geometry of the full model as the product of ordinary spacetime geometry and a finite geometry, both of these factor geometries being expressed most simply using quaternions.

Let us first give our conventions for charge conjugation in Euclidean space. For any representation of the Clifford algebra on $\mathbb{C}^{4}$, charge conjugation is given by the operator $R: \mathbb{C}^{4} \rightarrow \mathbb{C}^{4}$ defined by $R(v)=C^{-1} \mathbf{v}$, where the matrix $C$ satisfies $C \gamma^{\mu} C^{-1}=-\left(\gamma^{\mu}\right)^{T}, C^{T}=-C$ and $C \bar{C}=-1$. Also $\mathrm{C}_{\gamma} \mathrm{C}^{-1}=\bar{\gamma}$. The operator $\mathrm{R}$ preserves chirality.

Take $Q=C^{\infty}(Z) \otimes(\mathbb{C} \oplus \mathbb{H})$ and 
$3_{6}=\mathrm{L}^{2}(S) \otimes\left(\mathbb{C}^{2} \oplus \mathbb{C} \oplus \mathbb{C}^{2} \oplus \mathbb{C}^{2}\right) \otimes \mathbb{C}^{N_{G}}$. (The first $\mathbb{C}^{2}$ factor corresponds to $\left(e_{R}, d_{R}\right)$, the $\mathbb{C}$ factor corresponds to $u_{R}$, the second $\mathbb{C}^{2}$ factor corresponds to $\left(e_{L}, d_{L}\right)$ and the third $\mathbb{C}^{2}$ factor corresponds to $\left(v_{L}, u_{L}\right)$.) We will identify $\mathbb{H}$ with $\mathbb{C}^{2}$, where the action of an element $(\alpha, \beta)$ of $\mathbb{H}=$ $\mathbb{C}^{2}$ on $\mathbb{C}^{2}$ is given by a matrix $\left(\frac{\alpha}{-\beta} \frac{\beta}{\alpha}\right)$. The action of $(f,(\alpha, \beta)) \in Q$ on $\mathscr{H}$ is given by

$\left(\mathrm{fI}_{2} \oplus \mathrm{fI}_{1} \oplus\left(\begin{array}{cc}\alpha \mathrm{I}_{2} & \beta \mathrm{I}_{2} \\ \overline{-\beta} \mathrm{I}_{2} & \bar{\alpha} \mathrm{I}_{2}\end{array}\right)\right) \otimes \mathrm{I}_{\mathrm{N}_{\mathrm{G}}} \cdot$ The operator $\mathrm{D}$ is given by

$D(v)=\left(B \otimes\left(I_{2} \oplus I_{1} \oplus I_{2} \oplus I_{2}\right) \otimes I_{N_{G}}\right) v+$ $\gamma \otimes\left(\begin{array}{cccc}0 & 0 & 0 & 0 \\ 0 & 0 & 0 & -M_{2}^{T} \\ 0 & 0 & 0 & 0 \\ 0 & M_{2} & 0 & 0\end{array}\right) R(v)$

where $M_{1}=\left(\begin{array}{cc}M_{e} & 0 \\ 0 & M_{d}\end{array}\right)$ is a $2 N_{G} \times 2 N_{G}$ matrix and
$M_{2}=\left(\begin{array}{c}0 \\ M_{u}\end{array}\right)$ is a $2 N_{G} \times N_{G}$ matrix. $D$ is a symmetric operator with respect to the underlying real structure on $\mathscr{H}$. The $\mathbb{Z}_{2}$-grading is given by

$\Gamma=\gamma \otimes\left(\mathrm{I}_{2} \oplus \mathrm{I}_{1} \oplus\left(-\mathrm{I}_{2}\right) \oplus\left(-\mathrm{I}_{2}\right)\right) \otimes \mathrm{I}_{\mathrm{N}_{\mathrm{G}}}$

We will take the vector bundle $\&$ to be $G$. The unitary gauge group of $\varepsilon$ is $\operatorname{Map}(\mathrm{Z}, \mathrm{U}(1) \times \mathrm{SU}(2))$. The Hilbert space $E \otimes_{a} \mathscr{F}$ is the same as $\mathscr{F}$. A connection on $E$ is given by a skew element $\rho$ of $\Omega^{1}(a)$. A computation gives that $\pi(\rho)$ can be written as an operator on $\mathscr{H}$ in the form

$\pi(\rho)(v)=$

$\left(\begin{array}{cccc}A \otimes I_{N_{G}} & 0 & i\left(\bar{\phi}_{1}-1\right) \gamma \otimes M_{1}+i\left(\bar{\phi}_{2}\right) \gamma \otimes M_{1} \cdot \\ 0 & A \otimes I_{N_{G}} & 0 & 0 \\ i\left(\phi_{1}-1\right) \gamma \otimes M_{1} & 0 & A^{\prime} \otimes I_{N_{G}} & -W^{*} \otimes I_{N_{G}} \\ i \phi_{2} \gamma \otimes M_{1} & 0 & W \otimes I_{N_{G}} & -A^{\prime} \otimes I_{N_{G}}\end{array}\right) v$

$+\left(\begin{array}{cccc}0 & 0 & 0 & 0 \\ 0 & 0 & i \bar{\phi}_{2} \gamma \otimes M_{2}{ }^{T} & -i\left(\bar{\phi}_{1}-1\right) \gamma \otimes M_{2}{ }^{T} \\ 0 & -i \bar{\phi}_{2} \gamma \otimes M_{2} & 0 & 0 \\ 0 & \mathrm{i}\left(\bar{\phi}_{1}-1\right) \gamma \otimes M_{2} & 0 & 0\end{array}\right) R(v)$.

Â is a $U(1)$ gauge field, $\left(\begin{array}{cc}A^{\prime} & -W^{*} \\ W & -A^{\prime}\end{array}\right)$ is an SU(2) gauge field and $\Phi=\left(\begin{array}{l}\phi_{1} \\ \phi_{2}\end{array}\right)$ is a Higgs doublet with covariant derivative $\mathrm{D} \Phi=\mathrm{d} \Phi+\left(\begin{array}{cc}\mathrm{A}^{\prime}-\mathrm{A} & -\mathrm{W}^{*} \\ \mathrm{~W} & -\mathrm{A}^{\prime}-\mathrm{A}\end{array}\right) \Phi$. The image $\pi(\theta)$ can be written as an operator on $\mathscr{H}$ in the form

$\pi(\theta)(v)=S v+T \gamma R(v)$, where $S$ is a $4 \times 4$ self-adjoint matrix of operators with 


$$
\begin{aligned}
& S_{11}=F_{A} \otimes I_{N_{G}}+X \otimes I_{N_{G}}+\left(1-|\Phi|^{2}\right) \otimes M_{1}{ }^{\top} M_{1} \\
& s_{22}=F_{A} \otimes I_{N_{G}}+X \otimes I_{N_{G}}-\left(1-|\Phi|^{2}\right) \otimes M_{2}^{T} \overline{M_{2}}
\end{aligned}
$$

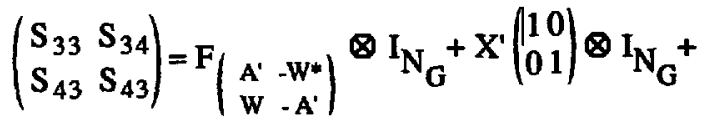

$$
\begin{aligned}
& 1 / 2\left(1-|\Phi|^{2}\right)\left(\begin{array}{ll}
1 & 0 \\
0 & 1
\end{array}\right) \otimes\left(M_{1} M_{1}^{\top}-M_{2} M_{2}^{\top}\right)+ \\
& \left(\begin{array}{cc}
\mathrm{Y} & \mathrm{Y}^{\prime *} \\
\mathrm{Y}^{\prime} & -\mathrm{Y}
\end{array}\right) \otimes\left[\mathrm{M}_{1} \mathrm{M}_{1}^{\top}+\mathrm{M}_{2} \mathrm{M}_{2}^{\dagger}\right] \\
& \left(\begin{array}{l}
S_{31} \\
S_{41}
\end{array}\right)=\mathrm{i}(\mathrm{D} \Phi) \gamma \otimes \mathrm{M}_{1}
\end{aligned}
$$

and $T$ is a $4 \times 4$ antisymmetric matrix of operators with

$\left(\begin{array}{l}T_{32} \\ T_{42}\end{array}\right)=i\left(\begin{array}{cc}0 & -1 \\ 1 & 0\end{array}\right) \overline{D \phi} \otimes M_{2}$

Here $X, X^{\prime}, Y$ and $Y^{\prime}$ are auxiliary scalar fields.

In order to provide the color, we will add an auxiliary algebra $\mathbb{B}=C^{\infty}(\mathrm{Z}) \oplus \mathrm{C}^{\infty}(\mathrm{Z})$ and a left $0 \mathrm{~s}$-module $\mathfrak{F}=C^{\infty}(Z) \oplus C^{\infty}(Z) \oplus C^{\infty}(Z) \oplus C^{\infty}(Z)=\mathbb{B}^{3}$ f, with

$f=\left(\begin{array}{ccc}(1,1) & 0 & 0 \\ 0 & (0,1) & 0 \\ 0 & 0 & (0,1)\end{array}\right)$. The gauge group of $\Im$ is $\operatorname{Map}(Z, U(1) \times U(3))$. Let us write the Hilbert space

$\mathscr{H}=L^{2}(S) \otimes\left(\mathbb{C}^{2} \oplus \mathbb{C} \oplus \mathbb{C}^{2} \oplus \mathbb{C}^{2}\right) \otimes \mathbb{C}^{N_{G}}$ as $\mathscr{H}_{6}=\mathscr{H}_{1} \oplus \mathscr{H}_{2} \oplus \mathfrak{H}_{3} \oplus \mathscr{H}_{4}$. We will let $\mathbb{B}$ act on the right on $\mathscr{H}_{1}, \mathscr{H}_{3}$ and $\mathscr{H}_{4}$, and on the left on $\mathscr{H}_{2}$. The action of $\left(g, g^{\prime}\right) \in \mathcal{B}$ on $\mathscr{F}_{1}, \mathscr{F}_{3}$ and $\mathscr{H}_{4}$ will be by $\left(\begin{array}{ll}g & 0 \\ 0 & g^{\prime}\end{array}\right)$ $\otimes \mathrm{I}_{\mathrm{N}_{\mathrm{G}}}$, and the action on $\mathfrak{H}_{2}$ will be by $\mathrm{gT} \mathrm{T}_{1} \otimes \mathrm{I}_{\mathrm{N}_{\mathrm{G}}} \cdot \mathrm{We}$ will then take the physical fermion space to be

$\left(\mathcal{F}_{1} \otimes_{B} \mathcal{F}\right) \oplus\left(\mathcal{F} * \otimes_{B} \mathcal{F}_{2}\right) \oplus\left(\mathcal{H}_{3} \otimes_{B} \mathcal{F}\right) \oplus\left(\mathcal{H}_{4}\right.$ $\left.\otimes_{B} \mathcal{F}\right)$.

This has the effect of adding the color to the quarks.

A connection on $\mathfrak{F}$ will give rise to a $U(1)$ gauge field and a $U(3)$ gauge field. The total gauge group is now $U(1)$ $x \operatorname{SU}(2) \times U(1) \times U(3)$. In order to reduce this to $U(1) \times$ SU(2) $\times$ SU(3), let us denote the $U(1)$ part of the connection on $\varepsilon$ by $-Y$. Let us require that the $U(1)$ part of the connection on $\mathcal{F}$ be - $Y$, and that the trace of the U(3) part of the connection on $F$ be $Y$. Upon computing the action as in section III, we obtain a Euclidean version of the standard model with $\mathrm{Y}$ as the hypercharge generator. We will discuss the possible relations among the coupling constants in section VI.

When written in Minkowski space, there are some slight differences due to the different properties of charge conjugation. Using the same matrices $\mathrm{C}$ and $\gamma$ as above, when we rotate to a $(+,-,-,-)$ Minkowski space the charge conjugation operator becomes $R(v)=\gamma^{0} C^{-1} \bar{v}$, which changes chirality. We will again take

$a=C^{\infty}(Z) \otimes(\mathbb{C} \oplus \mathbb{H})$ and $\mathscr{H}=L^{2}(S) \otimes\left(\mathbb{C}^{2} \oplus \mathbb{C} \oplus \mathbb{C}^{2} \oplus \mathbb{C}^{2}\right) \otimes \mathbb{C}^{N_{G}}$, with the same action of $C$ on $\mathscr{H}$. The operator $D$ will be given by $D(v)=\left(D \otimes\left(I_{2} \oplus I_{1} \oplus I_{2} \oplus I_{2}\right) \otimes I_{N_{G}}\right) v+$ 


$$
\begin{aligned}
& \text { i } \gamma \otimes\left(\begin{array}{cccc}
0 & 0 & M_{1} & 0 \\
0 & 0 & 0 & 0 \\
M_{1} & 0 & 0 & 0 \\
0 & 0 & 0 & 0
\end{array}\right) v+ \\
& i \gamma \otimes\left(\begin{array}{cccc}
0 & 0 & 0 & 0 \\
0 & 0 & 0 & M_{2}^{T} \\
0 & 0 & 0 & 0 \\
0 & M_{2} & 0 & 0
\end{array}\right) R(v) .
\end{aligned}
$$

(Our pure Dirac operator satisfies $D^{2}=-\partial_{0}^{2}+\partial_{j}^{2}$.) The $\mathbb{Z}_{2}$-grading will be given by

$$
\Gamma=\gamma \otimes\left(I_{2} \oplus\left(-I_{1}\right) \oplus\left(-I_{2}\right) \oplus\left(-I_{2}\right)\right) \otimes I_{N_{G}} \text {. Of the }
$$
various factors of $\mathfrak{H}$, the first $\mathbb{C}^{2}$ factor corresponds to $\left(e_{R}, d_{R}\right)$, the $\mathbb{C}$ factor corresponds to $u_{R} c$, the second $\mathbb{C}^{2}$ factor corresponds to $\left(e_{L}, d_{L}\right)$ and the third $\mathbb{C}^{2}$ factor corresponds to $\left(v_{L}, u_{L}\right)$. The rest of the discussion extends straightforwardly to Minkowski space. We will make the physical fermions chiral by requiring $\Gamma \psi=\psi$.

As with the previous models, it is possible to see our noncommutative spacetime geometry as the product of an ordinary spacetime geometry and the geometry over a point. We will do this for the electroweak geometry, for which it is convenient to use quaternions throughout and take tensor products over the quaternions. In the Euclidean case we use the fact that the spinor representation of Spin(4) is quaternionic, that is, the representation commutes with the generators of a quaternion algebra. This can be seen by noting that the double covering of the embeddings

$\mathrm{SO}(4) \rightarrow \mathrm{SO}(5) \rightarrow \mathrm{SO}(5,1)$ is
$(\operatorname{Spin}(4)=\mathrm{SU}(2) \times \mathrm{SU}(2)) \rightarrow \mathrm{U}(2, \mathbb{H}) \rightarrow \mathrm{SL}(2, \mathbb{H})$. The spinor bundle $S$ has fiber $\mathbb{H}^{2}$, and we will take the Clifford algebra to act on the right. Left multiplication by i defines a complex structure on $\mathbb{H}^{2}$, and the operator $L_{j}$ of left multiplication by $j$ then defines charge conjugation. $L_{j}$ is complex-antilinear, and because the chirality operator $\gamma$ is right multiplication by $\left(\begin{array}{cc}1 & 0 \\ 0 & -1\end{array}\right), \mathrm{L}_{\mathrm{j}}$ preserves chirality. The ordinary spacetime geometry is then given by

$a_{2}=C^{\infty}(Z)$ (a real algebra), $\mathscr{G}_{2}=L^{2}(S)$ with the right $a_{2}$ action,

$D_{2}=\left(\begin{array}{cc}0 & \partial_{0}+i \partial_{1}+j \partial_{2}+k \partial_{3} \\ -\partial_{0}+i \partial_{1}+j \partial_{2}+k \partial_{3} & 0\end{array}\right)$

(a real-symmetric operator acting on the right) and $\Gamma_{2}=\gamma$.

Over a point, we will take the algebra to be $\boldsymbol{Q}_{1}=\mathbb{C} \oplus \mathbb{H}$, where we will think of $\mathbb{C}=\mathbb{R} \oplus \mathbb{R}$ i as a subalgebra of $\mathbb{H}$. The (real) Hilbert space will be

$\mathscr{H}_{1}=\left(\mathbb{H}^{2} \oplus \mathbb{H}^{1} \oplus \mathbb{H}^{2} \oplus \mathbb{H}^{2}\right)^{N_{G}}$ with the action of

$(f, q) \in Q_{1}$ given by

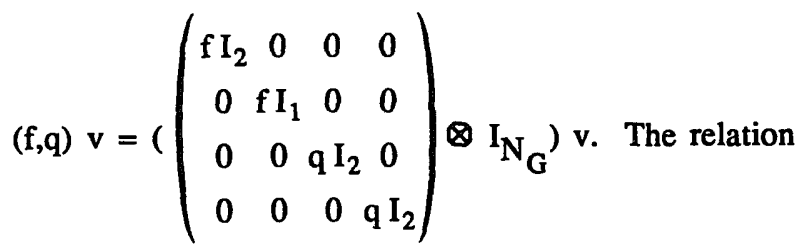
between this quaternionic description of the action of the algebra and the complex description is given by a change of basis, using the identity 


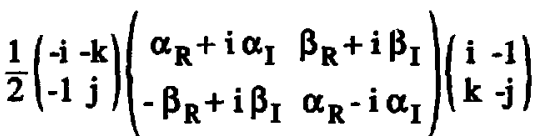

$=\left(\begin{array}{cc}\alpha_{R}+i \alpha_{I}+j \beta_{R}+k \beta_{I} & 0 \\ 0 & \alpha_{R}+i \alpha_{I}+j \beta_{R}+k \beta_{I}\end{array}\right)$.

The operator $D_{1}$ is $\left(\begin{array}{cc}0 & Z^{+} \\ Z & 0\end{array}\right)$, with

$Z=\left(\begin{array}{rr}-M_{1 I}+i M_{1 R} & M_{2 R}+i M_{2 I} \\ M_{1 R}+i M_{1 I} & -M_{2 I}+i M_{1 R}\end{array}\right)$

Here $M_{1 R}$ and $M_{1 I}$ are the real and imaginary parts of $M_{1}$, and similarly for $\mathrm{M}_{2}$.

We will take $\Gamma_{1}$ to be $\left(\begin{array}{cccc}I_{2} & 0 & 0 & 0 \\ 0 & I_{1} & 0 & 0 \\ 0 & 0 & -I_{2} & 0 \\ 0 & 0 & 0 & -I_{2}\end{array}\right) \otimes I_{N_{G}}$.

$D_{1}$ takes its particular form because it anticommutes with

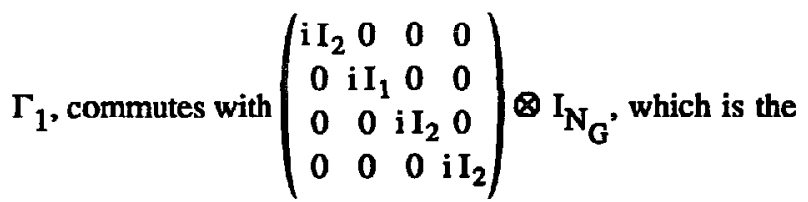
translation of the fact that in the complex description $\mathrm{D}$ acts complex linearly on the down quarks and complexantilinearly on the up quarks, and commutes with

$\left(\begin{array}{cccc}\mathrm{I}_{2} & 0 & 0 & 0 \\ 0 & -\mathrm{I}_{1} & 0 & 0 \\ 0 & 0 & 0 & \mathrm{i} \mathrm{I}_{2} \\ 0 & 0 & -\mathrm{i} \mathrm{I}_{2} & 0\end{array}\right) \otimes \mathrm{I}_{\mathrm{N}_{\mathrm{G}}}$, which is the translation of the fact that $D$ does not mix the up and down quarks.
The product geometry is ncw given by

$a=a_{1} \otimes a_{2}, r=H_{1} \otimes \otimes_{\text {H }}$,

$D=D_{1} \otimes \Gamma_{2}+I \otimes D_{2}$ and $\Gamma=\Gamma_{1} \otimes \Gamma_{2}$

In the Minkowski çase one can perform a similar construction using the fact that with signature $(+,-,-,-)$, the spinor representation is again quaternionic. This can be seen by noting that the double covering of the embeddings $S O(3,1) \rightarrow S O(4,1) \rightarrow S O(5,1)$ is

$(\operatorname{Spin}(3,1)=\operatorname{SL}(2, \mathbb{C})) \rightarrow \mathbf{U}(1,1, \mathbb{H}) \rightarrow \operatorname{SL}(2, \mathbb{H})$. The spinor bundle $S$ again has fiber $\mathbf{H}^{2}$, with the Clifford algebra acting on the right. Left multiplication by i defines a complex structure on $\mathrm{H}^{2}$, and the operator $\mathrm{L}_{\mathrm{j}}$ of left multiplication by $\mathbf{j}$ defines charge conjugation, a complexantilinear operator. The (antisymmetric) chirality operator $\gamma$ is right multiplication by $i$, which commutes with charge conjugation. The ordinary spacetime geometry is then given by

$a_{2}=C^{\infty}(\mathrm{Z})$ (a real algebra), $96_{2}=\mathrm{L}^{2}(\mathrm{~S})$ with the right $a_{2}$ action,

$D_{2}=\left(\begin{array}{rr}j \partial_{3}+k \partial_{4} & j \partial_{0}+j \partial_{1} \\ -j \partial_{0}+j \partial_{1} & j \partial_{3}+k \partial_{4}\end{array}\right)$

(a real operator acting on the right) and $\Gamma_{2}=\gamma$.

For the point geometry, we take the same $Q_{1}$ and $\mathscr{F}_{1}$ as in the Euclidean case, and we take $D_{1}=\left(\begin{array}{cc}0 & -Z^{4} \\ Z & 0\end{array}\right)$

$\Gamma_{1}=\left(\begin{array}{cccc}i I_{2} & 0 & 0 & 0 \\ 0 & -i I_{1} & 0 & 0 \\ 0 & 0 & 0 & I_{2} \\ 0 & 0 & -I_{2} & 0\end{array}\right) \otimes I_{N_{G}}$. The product geometry is given by 
$a=a_{1} \otimes a_{2}, \mathscr{G}=\mathscr{G}_{1} \otimes_{\mathbb{H}} \mathscr{H}_{2}$,

$D=D_{1} \otimes \Gamma_{2}+I \otimes D_{2}$ and $\Gamma=\Gamma_{1} \otimes \Gamma_{2}$.

\section{Discussion}

When one considers the renormalizability of our models, it will be important to know how restricted our actions are. First, it is possible that there are other operator-theoretically defined bosonic actions which reproduce the Yang-Mills action in the ordinary case. We will only consider here the simplest possibility, based on the Dixmier trace, as given in section II, (13). One can then consider two possibilities. The first uses the pure Dixmier trace to define the action, as in II (13). The second uses the fact that one can break $\mathrm{I}_{\nabla}$ into its various gauge-invariant pieces. That is, if $\left\{P_{i}\right\}$ are projections from $\mathbb{B B}\left(\Theta \otimes_{a} \mathscr{F}\right)$ to itself which satisfy $P_{i}\left(u_{T}{ }^{-1}\right)=P_{i}(T)$ for all $u \in U$ and $T \in B\left(\& \otimes_{Q} \mathscr{F}\right)$, then there is a gauge-invariant action given by

$I=\Sigma c_{i} \operatorname{Tr}_{\omega}\left(\left(P_{i}(\pi(\theta))\right)^{2} D_{\nabla}{ }^{-2 k}\right)$ for arbitrary constants $\left\{c_{i}\right\}$.

Let us illustrate this for the standard model of section V. Even if, to give the first possibility, we use the pure Dixmier trace, there is an arbitrary constant in front. For the standard model there are two distinct connections, coming from $\mathcal{E}$ and $\mathcal{F}$, and two unrelated Hilbert spaces corresponding to the leptons and quarks. There is no reason why the four ensuing Dixmier traces should be related, and so we have four arbitrary constants. This leads to one relation among the masses and coupling constants. In the limit in which the other fermion masses are negligible compared to the top quark mass, we find that the relation becomes that the Higgs mass is $2^{1 / 2}$ times the top mass. On the other hand, with the second possibility we find the same amount of arbitrariness for the constants as in the usual standard model.

If it turns out that the mass relation is satisfied, it might indicate that our action arises from a more unified operatortheoretic action. One could speculate that the special action obtained from a pure constant times the Dixmier trace is singled out among others as the restriction of an action with a much larger and simple invariance group of unitary operators.

We should stress two unsatisfactory features of our version of the standard model. The first is the need to relate the various $U(1)$ factors in order to get the right gauge group and hypercharge assignments. The second has to do with the different role played by the $B$ algebra and the chromodynamics sector. There may be some hint to the meaning of this from looking at what happens over a point in ordinary spacetime. There the role of the $\mathbb{B}$ algebra can be seen as a change of the ground ring $\mathbb{C}$ to $\mathbb{C} \oplus \mathbb{C}$, which is well understood as a feature of Kasparov's bivariant $\mathrm{KK}$ theory [Ka].

Let us note that as all of the fermion representations for our models arise from representations of algebras, the fermions must be in fundamental representations of the gauge groups. Thus it is impossible to get GUTs by our methods. We also need nonsimple gauge groups in order to have Higgs fields.

In conclusion, one way to look at this paper is the following. In the same way that Minkowski space arises naturally from Maxwell's theory, we look for the modification of spacetime arising from the electroweak unification. We want to find a theory on a (possibly) noncommutative spacetime which is comparable in simplicity to that of electrodynamics on an ordinary spacetime. Our noncommutative spacetime geometry can be considered as phenomenological, in that it is obtained unambiguously from the phenomenological action of the standard model. 
[Co1] A. Connes, "Noncommutative Differential Geometry", Publ. Math. IHES Vol. 62 (1986), p. 41

[Co2] A. Connes, "Essay on Physics and Noncommutative Geometry", in "The Interface of Mathematics and Particle Physics", eds. D. Quillen, G. Segal and S. Tsou, Oxford University Press (1990)

[Co3] A. Connes, "The Action Functional in Noncommutative Geometry", Comm. Math. Phys. 117 (1988), p.673

[Di] J. Dixmier, "Existence de Traces Non Normales", C.R. Acad. Sci. Paris 262 (1966), p. 1107

[EG] J. Ellis and M. Gaillard, Phys. Lett. B74 (1978), p. 374

[Ka] G. Kasparov, "Operator K-Theory and its Applications", Proc. Int. Cong. of Math., North-Holland, Amsterdam (1984), p. 987

[Gl] S. Glashow, Nucl. Phys. B22 (1961), p. 579

[PQ] R. Peccei and H. Quinn, Phys. Rev. D16 (1977), p. 1791

[Sa] A. Salam, Proc. 8th Nobel Symposium, ed. N. Svartholm, Almqvist and Wiksells, Stockholm (1968), p. 367

[We1] S. Weinberg, Phys. Rev. Lett. 19 (1967), p. 1264

[We2] S. Weinberg, Phys. Rev. Lett. 40 (1978), p. 223

[Wi] F. Wilczek, Phys. Rev. Lett. 40 (1978), p. 279 